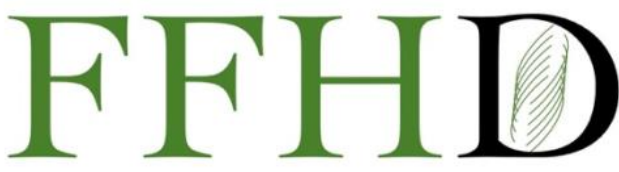

Functional Foods in Health and Disease

\title{
The effect of electron beam on oxidative stress and inflammatory factors in diabetes mellitus: An in vitro and in vivo study
}

\author{
Danik Martirosyan ${ }^{1}$, Masoomeh Shahnazari-Aval' ${ }^{2}$, Mohammad Reza Ashoori ${ }^{3}$, Afsaneh Seyed \\ Mikaeili ${ }^{4}$, Manouchehr Nakhjavani ${ }^{5}$ and Hossein Mirmiranpour ${ }^{5^{*}}$
}

${ }^{1}$ Functional Food Center, Functional Food Institute, Dallas, TX, USA; ${ }^{2}$ Department of Radio-Oncology, Cancer Institute, Valiasr Hospital, School of Medicine, Tehran University of Medical Science, Tehran, Iran; ${ }^{3}$ Department of Laboratory Sciences, School of Allied Medical Sciences, Zanjan University of Medical Sciences, Zanjan, Iran; ${ }^{4}$ Department of Molecular and Cellular Sciences, Faculty of Advanced Sciences and Technology, Islamic Azad University, Pharmaceutical Sciences Branch, Tehran, Iran; ${ }^{5}$ Endocrinology and Metabolism Research Center (EMRC), Valiasr Hospital, School of Medicine, Tehran University of Medical Science, Tehran, Iran

*Corresponding author: Hossein Mirmiranpour, MD, PhD, Endocrinology and Metabolism Research Center (EMRC), Valiasr Hospital, School of Medicine, Tehran University of Medical Science, Tehran, Iran.

Submission Date: April 13 ${ }^{\text {th }}$, 2021; Acceptance Date: July 6 ${ }^{\text {th }}, 2021$; Publication Date: July 21 ${ }^{\text {st }}, 2021$

Please cite this as: Martirosyan D., Shahnazari-Aval M., Ashoori MR., Mikaeili AS., Nakhjavani M., Mirmiranpour H. The effect of electron beam on oxidative stress and inflammatory factors in diabetes mellitus: An in vitro and in vivo study. Functional Foods in Health and Disease 2021. 11(7): 333-344. DOI: https://www.doi.org/10.31989/ffhd.v11i7.803

\section{ABSTRACT}

Background: The main purpose of this study was to investigate whether or not electron beam therapy (EBT) was an effective method in terms of moderating oxidative stress by reducing free radicals in BALB/c mice with type 1 diabetes mellitus.

Methods: The study was performed on thirty BALB/c mice in three groups including normal control, diabetic control, and EBT treated. Before studying the effect of electron beam on the studied groups, optimal level of constant source-to-surface distance, as well as the effects of EBT on glutathione reductase (GR) structure and function were determined. After studying the structure and the function of GR protein with three methods including fluorometry, circular dichroism (CD), and activity assay methods, SSD 100 was selected for EBT. Glucose, advanced glycation end-products, GR, oxidative stress factors such as hydrogen peroxide, malondialdehyde, 
advanced oxidation protein products, oxidized low-density lipoprotein, and inflammatory factors were measured in the serum of all groups.

Results: The results of in vitro study showed that electron beam therapy could increase glutathione reductase activity, which was not significant. Also, the results were compared between and within groups using one-way analysis of variance. Significant differences were observed for all variables measured between the normal control group and the other groups $(P<0.05)$. There was also no significant difference in blood glucose levels between the electron beam therapy treated group and the diabetic one $(P>0.05)$.

Diabetic Mice Treated with Electron Beam
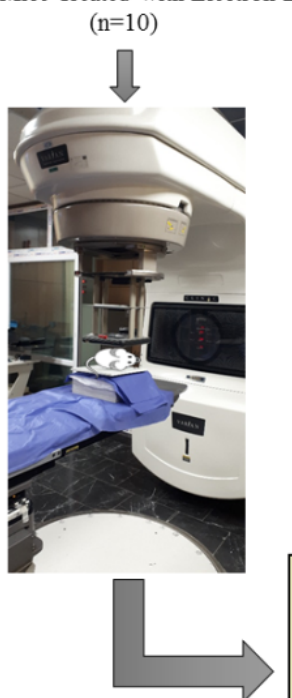

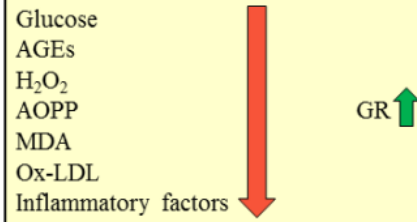

Normal Mice $(\mathrm{n}=10)$

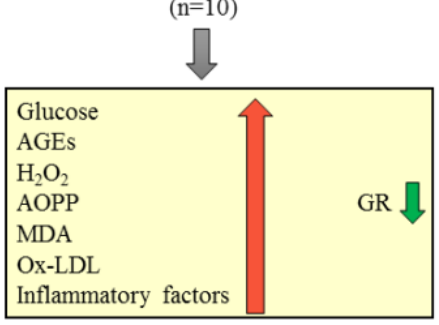

Glucose, AGEs, $\mathrm{H}_{2} \mathrm{O}_{2}$, AOPP, MDA

Ox-LDL, GR and inflammatory factors were normal

Conclusion: The results suggested that electron beam therapy could be effective in reducing free radicals and oxidative stress. Electron beam therapy, as a complementary method, might aid in moderating the complications of diabetes mellitus.

Keywords: Diabetes mellitus, Electron beam, Inflammatory factors, Oxidative stress

CFFC 2021. This is an Open Access article distributed under the terms of the Creative Commons Attribution 4.0 License (http://creativecommons.org/licenses/by/4.0)

\section{INTRODUCTION}

Diabetes mellitus (DM) (type 1 and type 2) is considered as one of the most prevalent chronic metabolic diseases [1]. Among the important risk factors for developing DM and its complications is insufficient physical activity [2-3]. It has been estimated that around 592 million people throughout the world will develop DM by the year 2035 [4]. In type 2 DM, also known as insulin-resistant DM, insulin is produced, but it loses its efficacy and results in insulin resistance [5]. Increased fasting plasma glucose (FPG), glycated hemoglobin (HbA1c), and 2-h 
plasma glucose levels are similarly known as diagnostic biomarkers of DM [6]. The production of oxidants, such as free radicals, in DM can also help with diagnosing pathogenesis and determining methods to treat it. Oxidative stress seems to be a major risk factor in the onset and progression of DM and its consequences [7]. Reactive oxygen species (ROS) also create oxidative stress. Moreover, hydrogen peroxide $\left(\mathrm{H}_{2} \mathrm{O}_{2}\right)$ is one of the most common ROS types which plays a role in hydroxyl radical $(\bullet \mathrm{OH})$ production, and $\bullet \mathrm{OH}$ has a position in the production of lipid peroxides [8]. Additionally, oxidative stress induces signaling pathways, contributing to immune and inflammatory responses [9]. Hyperglycemia with oxidative stress also plays an important role in the formation of advanced glycation end-products (AGEs) in patients with DM [10]. Accordingly, oxidative stress can be induced by AGEs [11], whose value in pathogenesis of DM and its consequences have not been fully understood [12]. Furthermore, evidence suggests that food-derived AGEs can contribute to development of DM and its complications [13-14].

Insulin-resistance and type 2 DM are identified by dyslipidemia and abnormal lipoproteins, such as oxidized low-density lipoprotein (Ox-LDL), which is a risk factor for DM consequences [15-16]. Ox-LDL and denatured LDL have also been isolated by AGEs with scavenger receptors, (SRs) which can form foam cells contributing to atherosclerosis [17]. Malondialdehyde (MDA) and advanced oxidation protein products (AOPP) are components of oxidative stress and are found more in individuals with diabetes compared to individuals without diabetes. MDA is a peroxidized degradation product of polyunsaturated fatty acids (PUFAs), and increased serum MDA along with higher levels of conjugated dienes have been observed in patients with DM [18]. In some studies, a positive correlation has been also reported between serum AOPP levels and $\mathrm{HbA} 1 \mathrm{c}$ concentration in patients with type 2 DM [19]. One of the causes of inflammation in the human body is inflammatory factors such as tumor necrosis factor alpha (TNF $\alpha$ ), interleukin 1 alpha (IL-1 $\alpha$ ), and interleukin 6 (IL-6), which are associated with insulin resistance and type 2 DM [20]. The mentioned inflammatory factors can increase in untreated type 1 and type 2 DM. Furthermore, it has been reported that acetoacetate as a ketone body increases secretion of TNF $\alpha$ and IL6 in hyper-ketonemic patients with DM [21].

It has also been found that oxidative stress affects microvascular and macrovascular diseases (as the complications of oxidative stress in type $2 \mathrm{DM}$ ) in the human body. Microvascular diseases contribute to the development of diabetic neuropathy and nephropathy. Reducing $\mathrm{HbA} 1 \mathrm{c}$ is also of importance in reducing microvascular events and consequences of neuropathy in patients with DM [22-23]. Antioxidants and some antioxidant enzymes such as glutathione reductase (GR) are able to break down free radicals and compounds created from free radicals. Using functional foods as a source of antioxidants can play an important role in preventing DM, as discussed in our previous study [24]. In untreated patients with DM, hyperglycemia leads to inhibition of antioxidant activity such as GR [25].

In most patients with type $1 \mathrm{DM}$, insulin is recommended for DM control. According to the American Diabetes Association (ADA), lifestyle changes can have effects on preventing and managing type 2 DM. Moreover, use of antihyperglycemic drugs can help in controlling type 2 DM and prevent its progression. In this regard, metformin is one of the most commonly used and recommended drugs for the management of type 2 DM [26]. Free radicals also have one or more unpaired electrons [27]. Therefore, elimination of 
free radicals and oxidative stress can be important in preventing DM consequences. The mechanism of action of electron therapy is related to its antioxidative effect. Electron therapy destroys free radicals [27]. Studies have reported the importance of EBT in the treatment and control of skin diseases and some cancers [28], as well as skin-related diseases caused by type 2 DM [29-30].There is no study on the application of EBT on free radicals and reduction of oxidative factors in diabetic samples, and this study could be a new study on the application of EBT.

The main purpose of this study was to evaluate the effect of EBT on oxidative stress and inflammatory factors, and to assess glucose and glutathione peroxidase levels in streptozotocin (STZ)-induced $\mathrm{BALB} / \mathrm{c}$ mice with diabetes.

\section{MATERIALS AND METHODS}

Materials: For the in vitro study, GR (G9297), glucose (G7021) and phosphate buffered saline (PBS) (P5368) were purchased from Sigma-Aldrich Co (USA). Activity assay kit of GR (ab83461) was also bought from Abcam Co (USA). Also, a $0.22 \mu \mathrm{m}$ filter was obtained from Millipore Corp., Billerica, MA (USA). For the in vivo study, STZ (S0130) and nicotinamide (NA) (N0636) were purchased from Sigma-Aldrich Co (USA). Moreover, quantity assay kits of IL1 $\beta$ (MBS175967), AGEs (MBS704846), AOPP (MBS263319), MDA (MBS264973) and ox-LDL (MBS2512757) were acquired from MyBioSource Co (USA). Quantity assay kits of IL1 $\alpha$ (BMS611), IL6 (LMC0061) and TNFa (BMC607-3) were bought from Termo Fisher Chem Co (USA). Also, quantity assay kits of $\mathrm{H}_{2} \mathrm{O}_{2}$ (E-BC-K102) and glucose (81692) were acquired from Elabscience and Crystal Chem companies (USA), respectively. It should be noted that EBT was performed by VARIAN model CLINAC 2100 C/D (USA).

\section{Methods}

GR Glycation: The method of glutathione reductase glycation was performed according to our previous study [31]. In this study, glucose solution was prepared via combining pure glucose with PBS. A solution of pure substance of GR, with a concentration of $10 \mathrm{mg} / \mathrm{ml}$, was also made through combining this protein with PBS at $\mathrm{pH}$ 7.4. Also, GR solution was prepared from pure GR solution. Then, a sample of the solution was mixed with glucose solution, with a concentration of $50 \mathrm{Mm} / \mathrm{lit}$, and named glycated GR solution. A part of this solution was directly separated and labeled with the same name, but the rest of the recently prepared solution was exposed to a physical factor (i.e. EBT) and named affected glycated GR. Therefore, there were three solutions; pure GR, glycated GR, and affected glycated GR. Following filtration of all samples under sterilized status, they were maintained in an incubator at $37^{\circ} \mathrm{C}$ for 16 weeks. At the end of the 16th week, an aliquot of each of the three previously mentioned solutions was prepared and then saved at $-80^{\circ} \mathrm{C}$ until it could be analyzed by fluorometry, circular dichroism (CD), and activity assay methods.

Optimal Constant Source-to-Surface Distance (SSD) Set up: Before examining the effects of EBT on oxidative and inflammatory stress factors in the study group and determining the optimal level of SSD, as well as the effects of EBT on GR structure and function, different SSDs and their impacts on pure protein, glycated protein, and glycated protein exposed to EBT were evaluated. After studying the structure and the function of GR protein in three mentioned statuses via fluorometry, CD, and activity assay methods, SSD 100 was selected for EBT. In fluorometry, the samples at a concentration of 0.5 $\mathrm{mg} / \mathrm{ml}$ were measured by Shimadzu 
Spectrofluorometer RF-5000 (Kyoto, Japan).

Excitation and emission wavelengths of 350 and 440 $\mathrm{nm}$ were also considered, respectively. The results were presented as percentages. In CD method, spectra assessment was done by JASCO-810 spectropolarimeter (Jasco, Tokyo, Japan). The structure of each of the previously mentioned samples containing a concentration of $0.1 \mathrm{mg} / \mathrm{ml}$ was also measured. The spectra were modulated and achieved as units of mean residue molar ellipticity $[\theta]$ (mdeg $\mathrm{cm}^{2} \mathrm{dmol}^{-1}$ ), based on the average weight of the amino acids (112.4). Moreover, the equation [ $\theta$ ] $\lambda=(\theta \times 112.4) / \mathrm{cl}$ showed the molar ellipticity and calculations were performed at $25^{\circ} \mathrm{C}$. Furthermore, the function of GR was measured by a related activity assay kit (enzyme-linked immunosorbent assay method (ELISA)), enzymatic colorimetric method, and Tecan's Sunrise absorbance microplate reader (Switzerland). The measurement of enzyme activity was performed as $\mathrm{mU} / \mathrm{ml}$.

Animals: A total number of 40 male BALB/c-type mice aged 6 weeks with an average body weight of $30 \mathrm{~g}$ (bought from Pasteur Institute, Tehran, Iran) were housed in a temperature-controlled vivarium (at a temperature of $23 \pm 3^{\circ} \mathrm{C}$ and a relative humidity of $50 \pm 10 \%)$ with a $12: 12 \mathrm{~h}$ light-dark cycle and free access to rat chow and water ad libitum. The mice were given a normal diet. The experimental protocol was approved by the Animal Ethical Committee in accordance with the guidelines for the care and use of laboratory animals prepared by Tehran University of Medical Sciences. After one week of acclimation under these conditions, 30 mice showing favorable growth were selected and used for further studies. They were randomly allocated to three groups $(n=10)$ : (1) normal control group (normal ones without any interference), (2) diabetic control group (with diabetes ones involved by STZ but not affected by EBT), (3) EBT treated group (with diabetes ones affected by EBT). DM was then induced in mice (groups 2 and 3) via intraperitoneal injection (i.p) of a single dose of STZ (50 mg/kg) 15 min after an i.p administration of nicotinamide $(120 \mathrm{mg} / \mathrm{kg})$. One week after such injections, serum glucose $\geq 200 \mathrm{mg} / \mathrm{dl}$ was considered as DM. Hence, mice were divested of food for 10-12 h. Blood samples $(20 \mu \mathrm{l})$ were also collected from mouse tail veins under ether anesthesia. Group 3 was then exposed to EBT after induction of DM owing to STZ. The experiments were carried out in compliance with the relevant national laws relating to the conduct of animal experimentation.

EBT: In the present study, EBT was performed using a linear accelerator. Before exposure to electron beams, mice were divested of food for $10-12 \mathrm{~h}$ and then were anesthetized with xylazine and ketamine. The dose for per treat in all mice was $100 \mathrm{cGY}$ of energy. Treatment was also performed in two fractions (one fraction twice in two weeks). The used amount of energy was $9 \mathrm{MeV}$ (micro electron volt). The depth of treatment was $1.5 \mathrm{~cm}$. After the EBT was completed, mice in all three groups were sacrificed following anesthesia. Then, blood samples were taken from their hearts. To separate the serum from the clot, the clot was centrifuged for $5 \mathrm{~min}$ at $5000 \mathrm{~g}$. The isolated sera were then stored at $-70^{\circ} \mathrm{C}$ for further study. Serum glucose in all groups was subsequently measured according to the glucose kit instructions.

Statistical analysis: Statistical analyses were performed using SPSS for Microsoft Windows (version 16.0; IBM, USA). The results were thus shown as means \pm standard deviation (SD). The mean scores of the variables between and within groups were also 
compared using one-way analysis of variance (ANOVA). After that, Tukey's honestly significant difference (HSD) post-hoc test for multiple comparisons was employed. To analyze the in vitro results, Mann-Whitney $U$ test, as a nonparametric test, was utilized. A p-value $\leq 0.05$ characterized the presence of a statistically significant difference.

\section{RESULTS}

In Vitro Study: As mentioned before, the activity of GR protein in a glycated state was investigated to determine optimal levels of SSDs and the impact of different SSDs on the results of EBT. After establishing the optimal SSD (i.e. SSD $=100$ ) activity and concentration, the structure of GR in glycated and electron-affected states was investigated by three methods. In addition to measuring the activity and structure of GR under the influence of EBT, the activity of this protein in pure and glycated states was also studied. GR structure, concentration, and activity assays were performed in three different modes-by $C D$, fluorometry and ELISA (according to the manufacturer's instructions) (Table 1). Evaluation was done in triplicate and results indicated a significant difference in concentration and structure of glycated GR and glycated GR exposed to EBT (pvalue $<0.05)$. Comparison of GR activity between the two groups showed no significant difference ( $p$-value $>0.05)$.

Table 1. Activity, concentration, and structure of GR in three forms, pure, glycated and glycated treated with electron radiation.

\section{Method type}

\begin{tabular}{llll} 
Samples & Fluorometry $(\%)$ & Circular dichroism $(\boldsymbol{\theta} / \mathrm{nm})$ & ELISA $^{*}\left(\mathrm{mU} / \mathrm{ml}^{\prime}\right)$ \\
\hline Pure GR & $23.75 \pm 0.7$ & $195248 \pm 3.64$ & $32.5 \pm 1.28$ \\
\hline Glycated GR & $100 \pm 1.63$ & $331267 \pm 4.19$ & $21.7 \pm 1.06$ \\
\hline Glycated GR exposed to electron radiation & $91.36 \pm 1.32$ & $326475 \pm 4.93$ & $23.6 \pm 1.08$ \\
\hline P-value** & $<0.001$ & $<0.001$ & $<0.001$
\end{tabular}

GR, glutathione reductase. Data are expressed as mean \pm SD. SSD for the sample treated with electron was 100

*The enzyme-linked immunosorbent assay

**Significant difference between pure GR and glycated GR exposed to electron radiation

In Vivo Study: After the diabetic group was exposed to electron beams (Figure 1), the mice of all groups were sacrificed and the mentioned above biochemical parameters were examined. There was a significant difference between blood glucose levels in the diabetic control group and the EBT treated group $(p$-value $=0.001)$. However, there was no significant difference in blood glucose levels between diabetic control group and EBT treated group ( $p$-value $=0.84)$. In addition to glucose, serum GR, AGEs, and oxidative factors were measured in all groups, and a significant difference was observed between the normal control group and the other groups. Comparison of these factors between the EBT treated group and diabetic control group was also significant ( $p$-value $=0.001)$. These results are presented in Table 2. 


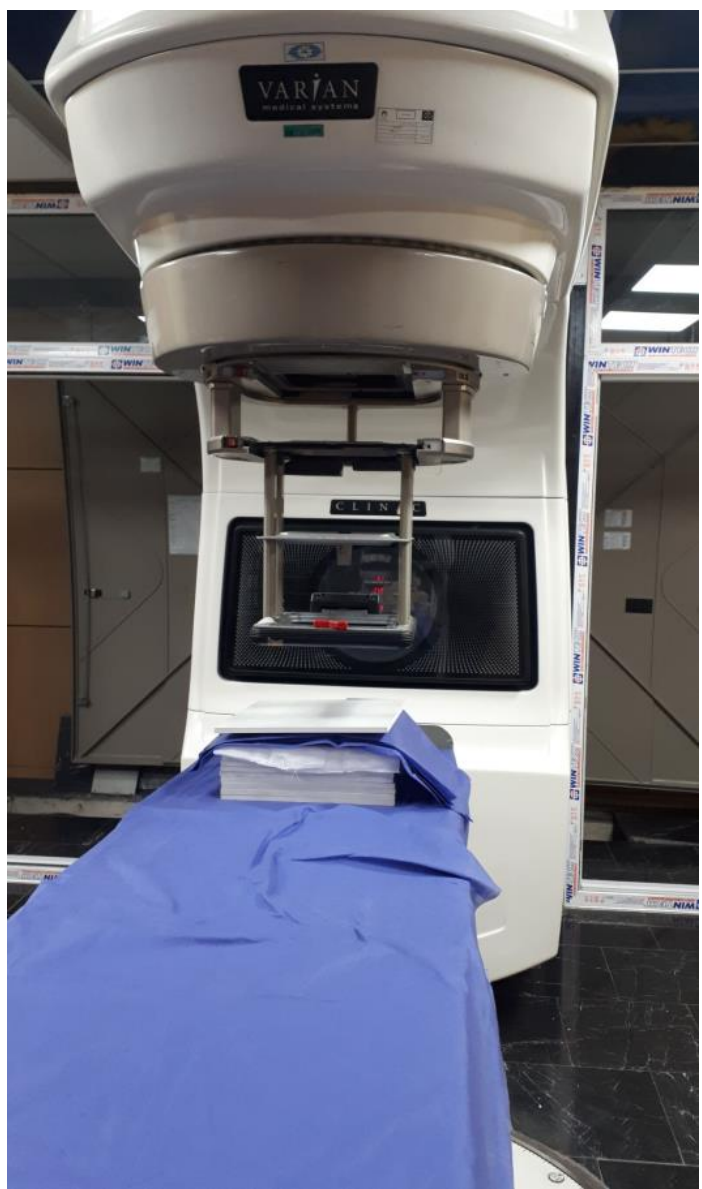

Figure 1. Scheme of the radiotherapy system and the diabetic group were treated with electron beams (EBT group)

Table 2. Comparison between serum glucose concentration and other measured variables in different studied groups

\begin{tabular}{|c|c|c|c|}
\hline Groups Parameters & Normal control $(n=10)$ & Diabetic control $(n=10)$ & EBT treated $(n=10)$ \\
\hline Glucose (mg/dl) & $163.70 \pm 2.90^{*}$ & $399.60 \pm 22.72$ & $395.60 \pm 15.70$ \\
\hline $\mathrm{GR}(\mathrm{mU} / \mathrm{ml})$ & $33.17 \pm 1.26^{*}$ & $21.24 \pm 1.54$ & $24.04 \pm 0.95^{+}$ \\
\hline AGEs $(\mu \mathrm{g} / \mathrm{ml})$ & $5.37 \pm 0.17^{*}$ & $9.20 \pm 0.25$ & $8.65 \pm 0.26^{\dagger}$ \\
\hline $\mathrm{H}_{2} \mathrm{O}_{2}(\mathrm{mmol} / \mathrm{l})$ & $31.88 \pm 2.76^{*}$ & $131.94 \pm 2.42$ & $127.69 \pm 1.45^{+}$ \\
\hline AOPP (mg/ml) & $17.64 \pm 0.80^{*}$ & $42.82 \pm 1.73$ & $38.25 \pm 2.38^{+}$ \\
\hline MDA (nmol/ml) & $5.10 \pm 0.32^{*}$ & $9.09 \pm 0.18$ & $8.69 \pm 0.42^{\dagger}$ \\
\hline Ox-LDL (ng/ml) & $8.04 \pm 0.36^{*}$ & $18.46 \pm 0.26$ & $18.01 \pm 0.37^{\dagger}$ \\
\hline
\end{tabular}

GR, glutathione reductase; AGEs, advanced glycation end-products; AOPP, advanced oxidation protein products; MDA, malondialdehyde; Ox-LDL, Oxidized low-density lipoprotein. Results are expressed as the mean \pm SD.

* Significances of data comparing in the normal control group with the other of groups ( $P$ value $<0.001)$.

+ Significances of data comparing EBT treated vs. diabetic control group ( $P$ value $<0.05)$. 
In this study, inflammatory factors, which may be elevated in the blood as a result of untreated DM, were also evaluated. Comparison of inflammatory factors such as IL-1 $\alpha, \mathrm{IL}-1 \beta, \mathrm{IL}-6$, and TNF $\beta$ showed a significant difference $(p$-value $=0.001$ ) between the normal control group with other groups and the diabetic control group with the EBT treated group (Figure 2).

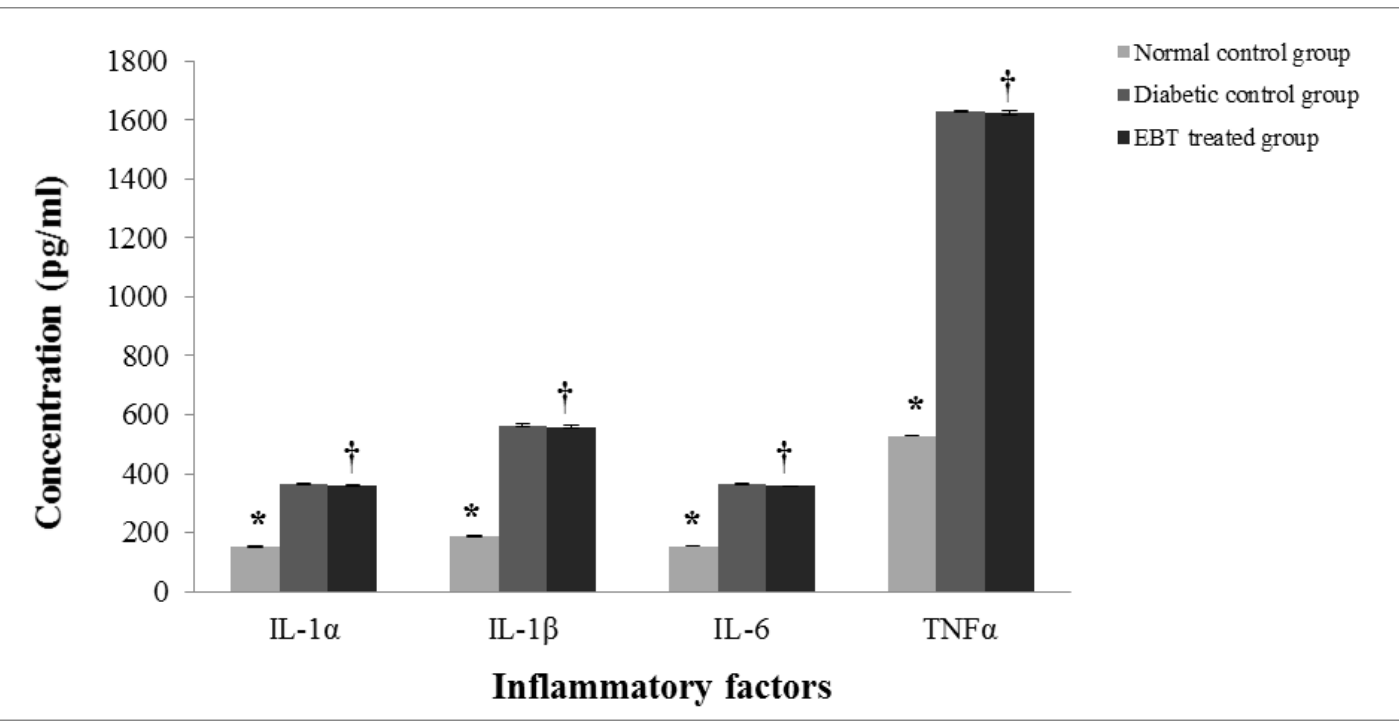

Figure 2. Concentration of IL-1 $\alpha, I L-1 \beta, I L-6$ and TNF $\alpha$ in the normal control group, diabetic control group and EBT treated group.

* Significances of data comparing normal control group vs. other groups

+ Significances of data comparing EBT treated group vs. diabetic control group

\section{DISCUSSION}

The main purpose of this study was to investigate the effects of EBT on serum glucose and some oxidative stress and inflammatory factors in mice with type 1 DM. Consequences of untreated DM are one of the most important issues in human communities. Accordingly, oxidative stress and free radicals, as well as some inflammatory factors caused by DM, are involved in the formation of DM consequences [32], and it is clear that untreated DM increases the variables of oxidative stress and inflammatory factors. These variables have already been investigated in numerous studies and it has been suggested that some elements play an important role in reducing them [33-40]. The pro-inflammatory mechanisms can also be of importance in the development of diabetic nephropathy; e.g. in the nuclear factor kappa-light-chain-enhancer of activated $B$ cells (NFKB)-TNF $\alpha$ pathway, TNF $\alpha$ is stimulated by increased oxidative stress as well as glycated and oxidized products. This inflammatory factor in untreated DM similarly plays a leading role in the development of diabetic nephropathy [41].

Radiation therapy and laser therapy are now being utilized to treat patients with cancer. However, no study so far has investigated the effects of EBT on free radicals and inflammatory factors in patients with diabetes, although there have been investigations into laser therapy in patients with DM as well as animal models [42-45]. Radiation therapy may be harmful and can also lead to DM. In this respect, one study found that pancreas exposure to 
radiation might increase the risk of DM in children with cancer [46]. In a study by Enosawa et al., serum glucose and malondialdehyde levels were reduced in electron-treated diabetic rats in the twelfth week [47]. The results of serum levels of glucose and malondialdehyde in our study were consistent with the results of the study of Enosawa et al. A study by Skrepnik et al. also showed that long-term EBT was useful for the treatment of skin lesions (i.e. scleroderma diabeticorum) caused by poorly controlled DM [30].

In the present study, the penetration depth of the electron beam was $1.5 \mathrm{~cm}$, which might affect the skin and subcutaneous blood flow. Electron beams do not penetrate deep into the body and do not affect the tissues and organs of the body. It is possible that free radicals with the electrons given will complete their capacity and lose their oxidizing power. Probably for this reason, in the present study, the amount of $\mathrm{H}_{2} \mathrm{O}_{2}$ had been decreased in the mice in the EBT treated group compared to the untreated group. This might also be the reason for the decrease in OxLDL, AOPP, and MDA in EBT treated mice. The AGEs can be correspondingly involved in the development of diabetes. The production of AGEs occurs in hyperglycemic and oxidative stress conditions. It has been previously demonstrated in our study that the laser in vitro affects glycated catalase [31]. Electron irradiation may also directly or indirectly reduce glycated compounds such as AGEs at the serum level. In this regard, EBT can be a new method to reduction of glycated compounds. Free radicals can thus destruct antioxidant enzymes. By reducing free radicals, the activity of antioxidant enzymes such as catalase and GR can subsequently increase. In this study, minerals and vitamins were not measured, but in our previous studies $[24,48]$, minerals were studied and the effect of using functional foods on the increase of these elements and oxidative biomarkers [49] in diabetic samples was discussed. The growth in GR activity in the serum of mice treated with EBT confirms the results of this in vitro study, in which it was found that the activity of glycated GR treated with EBT had increased more than glycated GR. However, this increasing trend was not significant. In the present study, the small sample size was the most important limitation and larger sample size is required to verify these results.

\section{CONCLUSION}

In conclusion, EBT can be used as a complementary treatment to avoid the complications of conventional DM medications such as insulin and metformin. It can be stated that EBT may be involved in reducing some free radicals and oxidative stress caused by DM. Further studies, however, need to be carried out both in vitro and in vivo.

List of abbreviations: EBT: electron beam therapy, DM: diabetes mellitus, WHO: World Health Organization, FPG: fasting plasma glucose, HbA1c: glycated hemoglobin, ROS: Reactive oxygen species, $\mathrm{H}_{2} \mathrm{O}_{2}$ : hydrogen peroxide, AGEs: advanced glycation end-products, Ox-LDL: oxidized low-density lipoprotein, MDA: Malondialdehyde, AOPP: advanced oxidation protein products, TNFa: tumor necrosis factor alpha, IL-1 $\alpha$ : interleukin 1 alpha, IL-6: interleukin 6, GR: glutathione reductase, PBS: phosphate buffered saline, CD: circular dichroism, SSD: Source-to-Surface Distance, ELISA: enzymelinked immunosorbent assay.

Author's contributions: The authors' confirmed contributions to the paper are as follows: DM 
participated in the study design and the article editing. MSA performed experiments and treatment of samples with cold plasma by atmospheric pressure plasma jet device; MRA participated in the writing and analysis of the results; ASM assisted in experiments; $\mathrm{MN}$ and $\mathrm{HM}$ contributed to the original idea of the paper, doing the experimental work and data collection. All authors read and approved the final version before its submission.

Conflict of interest: The authors declare that there is no conflict of interest.

Acknowledgments: The authors of this manuscript would like to thank the Endocrinology Research Center of Tehran University of Medical Sciences for their financial support. The authors of this article acknowledge also the staff of the Valiasr Hospital Cancer Institute.

\section{REFERENCES}

1. Ashoori MR, Rahmati-Yamchi M, Ostadrahimi $A$ Pahlavan-Gharebaba R, Mobasseri M, Bakhtiyari S, et al.: Apelin-13 serum levels in type 2 diabetic obese women: possible relations with microRNAs-107 and 375. Turk Biyokim Derg 2019, 44 (5):667-675.

2. Gambineri A, Pelusi C: Sex hormones, obesity and type 2 diabetes: is there a link? Endocr Connect 2019, 8 (1):R1-R9.

3. Ashoori MR, Rahmati-Yamchi M, Ostadrahimi A, Aval SF Zarghami N: MicroRNAs and adipocytokines: promising biomarkers for pharmacological targets in diabetes mellitus and its complications. Biomed Pharmacother 2017, 93 1326-1336.

4. DeFronzo RA, Ferrannini E, Groop L, Henry RR, Herman WH, Holst JJ, et al.: Type 2 diabetes mellitus. Nat Rev Dis Primers 2015, 115019.

5. Kahn BB, Flier JS: Obesity and insulin resistance. J Clin Invest 2000, 106 (4):473-481.

6. Sacks DB, Arnold M, Bakris GL, Bruns DE, Horvath AR, Kirkman MS, et al.: Guidelines and recommendations for laboratory analysis in the diagnosis and management of diabetes mellitus. Clin chem 2011, 57 (6):e1-e47

7. Rains JL, Jain SK: Oxidative stress, insulin signaling, and diabetes. Free Radic Biol Med 2011, 50 (5):567-575.

8. Tangvarasittichai S: Oxidative stress, insulin resistance, dyslipidemia and type 2 diabetes mellitus. World J Diabetes 2015, 6 (3):456.

9. Bloch-Damti A, Bashan N: Proposed mechanisms for the induction of insulin resistance by oxidative stress. Antioxid Redox Signal 2005, 7 (11-12):1553-1567.

10. Wright Jr E, Scism-Bacon J, Glass L: Oxidative stress in type 2 diabetes: the role of fasting and postprandial glycaemia. Int J Clin Pract 2006, 60 (3):308-314.

11. Brownlee $\mathrm{M}$ : The pathobiology of diabetic complications: a unifying mechanism. diabetes 2005, 54 (6):1615-1625

12. Nowotny K, Jung T, Höhn A, Weber D, Grune T: Advanced glycation end products and oxidative stress in type 2 diabetes mellitus. Biomolecules 2015, 5 (1):194222.

13. Uribarri J, Cai W, Peppa M, Goodman S, Ferrucci L, Striker G, et al.: Circulating glycotoxins and dietary advanced glycation endproducts: two links to inflammatory response, oxidative stress, and aging. J Gerontol A Biol Sci Med Sci 2007, 62 (4):427-433.

14. Cai W, Ramdas M, Zhu L, Chen X, Striker GE, Vlassara H: Oral advanced glycation endproducts (AGEs) promote insulin resistance and diabetes by depleting the antioxidant defenses AGE receptor-1 and sirtuin 1. Proc Natl Acad Sci 2012, 109 (39):15888-15893.

15. Grundy SM: Hypertriglyceridemia, atherogenic dyslipidemia, and the metabolic syndrome. Am J Cardiol 1998, 81 (4):18B-25B.

16. Taskinen M-R: Diabetic dyslipidaemia: from basic research to clinical practice. Diabetologia 2003, 46 (6):733-749.

17. Takenaka K, Yamagishi S-i, Matsui T, Nakamura K, Imaizumi T: Role of advanced glycation end products (AGEs) in thrombogenic abnormalities in diabetes. Curr Neurovasc Res 2006, 3 (1):73-77.

18. Pan H-z, Zhang L, Guo M-y, Sui H, Li H, Wu W-h, et al.: The oxidative stress status in diabetes mellitus and diabetic nephropathy. ACTA DIABETOL 2010, 47 (1):7176.

19. Pandey KB, Mishra N, Rizvi SI: Protein oxidation biomarkers in plasma of type 2 diabetic patients. Clin biochem 2010, 43 (4-5):508-511. 
20. Freitas Lima LC, Braga VdA, do Socorro de França Silva M, Cruz JdC, Sousa Santos SH, de Oliveira Monteiro MM, et al.: Adipokines, diabetes and atherosclerosis: an inflammatory association. Front Physiol 2015, 6304.

21. Jain SK, Kannan K, Lim G, Matthews-Greer J, McVie R, Bocchini JA: Elevated blood interleukin-6 levels in hyperketonemic type 1 diabetic patients and secretion by acetoacetate-treated cultured U937 monocytes. Diabetes Care 2003, 26 (7):2139-2143.

22. Fowler MJ: Microvascular and macrovascular complications of diabetes. Clin diabetes 2008, 26 (2):7782.

23. Folli F, Corradi D, Fanti P, Davalli A, Paez A, Giaccari A, et al.: The role of oxidative stress in the pathogenesis of type 2 diabetes mellitus micro-and macrovascular complications: avenues for a mechanistic-based therapeutic approach. Curr Diabetes Rev 2011, 7 (5):313-324.

24. Martirosyan D, Ashoori MR, Mirmiranpour H: The effect of low level-laser irradiation on antioxidant enzymes and mineral levels in serum of patients with type 2 diabetes mellitus. Bioact Compd Health Dis 2020, 3 (5):82-89

25. Aouacheri O, Saka S, Krim M, Messaadia A, Maidi I: The investigation of the oxidative stress-related parameters in type 2 diabetes mellitus. Can J Diabetes 2015, 39 (1):44-49.

26. Perriello G, Misericordia P, Volpi E, Santucci A, Santucci C) Ferrannini $E$, et al.: Acute antihyperglycemic mechanisms of metformin in NIDDM: evidence for suppression of lipid oxidation and hepatic glucose production. Diabetes 1994, 43 (7):920-928.

27. Poprac P, Jomova K, Simunkova M, Kollar V, Rhodes CJ, Valko $M$ : Targeting free radicals in oxidative stressrelated human diseases. Trends Pharmacol Sci 2017, 38 (7):592-607.

28. Navi D, Riaz N, Levin YS, Sullivan NC, Kim YH, Hoppe RT: The Stanford University experience with conventionaldose, total skin electron-beam therapy in the treatment of generalized patch or plaque (T2) and tumor (T3) mycosis fungoides. Arch Dermatol 2011, 147(5):561567.

29. Kroeger K, Elsayad K, Moustakis C, Haverkamp U, Eich HT: Low-dose total skin electron beam therapy for cutaneous lymphoma. Strahlenther Onkol 2017, 193 (12):1024-1030.
30. Skrepnik T, Gottesman S, Stea B: Long-term benefit of electron beam radiation therapy in the treatment of scleredema of Buschke. Adv Radiat Oncol 2016, 1 (1):2125.

31. Mirmiranpour H, Nosrati FS, Sobhai SO, Takantape SN, Amjadi A: Effect of Low-Level Laser Irradiation on the Function of Glycated Catalase. J Lasers Med Sci 2018, 9 (3):212.

32. Kayama Y, Raaz U, Jagger A, Adam M, Schellinger I, Sakamoto M, et al.: Diabetic cardiovascular disease induced by oxidative stress. Int J Mol Cell Med 2015, 16 (10):25234-25263

33. Phosat C, Panprathip P, Chumpathat N, Prangthip $P$, Chantratita N, Soonthornworasiri N, et al.: Elevated Creactive protein, interleukin 6 , tumor necrosis factor alpha and glycemic load associated with type 2 diabetes mellitus in rural Thais: a cross-sectional study. BMC Endocr Disord 2017, 17 (1):44.

34. Samarghandian S, Azimi-Nezhad M, Farkhondeh $\mathrm{T}$ : Crocin attenuate Tumor Necrosis Factor-alpha (TNF- $\alpha$ ) and interleukin-6 (IL-6) in streptozotocin-induced diabetic rat aorta. Cytokine 2016, 88 20-28.

35. Kousathana F, Georgitsi M, Lambadiari V, GiamarellosBourboulis EJ, Dimitriadis G, Mouktaroudi M: Defective production of interleukin-1 beta in patients with type 2 diabetes mellitus: restoration by proper glycemic control. Cytokine 2017, 90 177-184.

36. Anquetil F, Sabouri S, Thivolet C, Rodriguez-Calvo T, Zapardiel-Gonzalo J, Amirian N, et al.: Alpha cells, the main source of IL-1 $\beta$ in human pancreas. J Autoimmun 2017, 81 68-73.

37. Rodrigues KF, Pietrani NT, Bosco AA, Campos FMF, Sandrim VC, Gomes KB: IL-6, TNF- $\alpha$, and IL-10 levels/polymorphisms and their association with type 2 diabetes mellitus and obesity in Brazilian individuals. Arch Endocrinol Metab 2017, 61 (5):438-446.

38. Venturini D, Simão ANC, Dichi I: Advanced oxidation protein products are more related to metabolic syndrome components than biomarkers of lipid peroxidation. Nutr Res 2015, 35 (9):759-765.

39. Ganjifrockwala F, Joseph J, George G: Serum oxidized LDL levels in type 2 diabetic patients with retinopathy in Mthatha Region of the Eastern Cape Province of South Africa. Oxid Med Cell Longev 2016, 2016

40. Panahi $Y$, Khalili N, Sahebi E, Namazi S, Karimian MS, Majeed $M$, et al.: Antioxidant effects of curcuminoids in patients with type 2 diabetes mellitus: a randomized 
controlled trial. Inflammopharmacology 2017, 25

(1):25-31.

41. Cameron NE, Cotter MA: Pro-Inflammatory Mechanisms in Diabetic Neuropathy: Focus on the Nuclear Factor Kappa B Pathway. Curr Drug Targets 2008, 9 (1):60-67.

42. Elman MJ, Aiello LP, Beck RW, Bressler NM, Bressler SB, Edwards AR, et al.: Randomized trial evaluating ranibizumab plus prompt or deferred laser or triamcinolone plus prompt laser for diabetic macular edema. Ophthalmology 2010, 117 (6):1064-1077. e1035.

43. Amjadi A, Mirmiranpour H, Sobhani SO, Goudarzi NM: Intravenous laser wavelength radiation effect on LCAT, PON1, catalase, and FRAP in diabetic rats. Laser Med Sci 2019, 1-8.

44. Tatmatsu-Rocha JC, Ferraresi C, Hamblin MR, Maia FD, do Nascimento NRF, Driusso $P$, et al.: Low-level laser therapy $(904 \mathrm{~nm})$ can increase collagen and reduce oxidative and nitrosative stress in diabetic wounded mouse skin. J Photochem Photobiol B 2016, 164 96-102.

45. Kazemikhoo N, Sarafnejad A, Ansari F, Mehdipour P: Modifying effect of intravenous laser therapy on the protein expression of arginase and epidermal growth factor receptor in type 2 diabetic patients. Lasers Med Sci 2016, 31 (8):1537-1545.

46. Friedman DN, Moskowitz CS, Hilden P, Howell RM, Weathers RE, Smith SA, et al.: Radiation Dose and Volume to the Pancreas and Subsequent Risk of Diabetes Mellitus. J Natl Cancer Inst 2019,

47. Enosawa S, Dozen M, Tada $\mathrm{Y}$, Hirasawa K: Electron therapy attenuated elevated alanine aminotransferase and oxidative stress values in type 2 diabetes-induced nonalcoholic steatohepatitis of rats. Cell medicine 2013, $6(1-2): 63-73$.

48. Martirosyan D, Ghomi H, Ashoori MR, Rezaeinezhad A, Mirmiranpour $\mathrm{H}$ : The effect of cold plasma on antioxidant enzymes, minerals, and some of the levels of the biochemical parameters in the subjects with type 2 diabetes mellitus samples. Bioact Compd Health Dis 2021, 4 (2):14-24.

49. Martirosyan D, Mirmiranpour H, Ashoori MR: Synergistic effect of laser irradiation and cinnamic acid as a functional food on oxidative stress in type 2 diabetes mellitus. Bioact Compd Health Dis 2020, 3 (9):154-165. 